\title{
BOREL'S FIXED POINT THEOREM FOR KAEHLER MANIFOLDS AND AN APPLICATION
}

\author{
ANDREW J. SOMMESE
}

\begin{abstract}
A short proof of a generalization of the Borel fixed point theorem to the case of Kaehler manifolds is given and, as an application, a short proof of Wang's theorem that compact simply connected homogeneous manifolds are projective and of the form $G / P$, where $G$ is a complex semisimple Lie group and $P$ is a parabolic subgroup.
\end{abstract}

I will give a short proof of a generalization of the Borel fixed point theorem to the case of Kaehler manifolds and, as an application, give a short proof of Wang's theorem that compact simply connected homogeneous Kaehler manifolds are projective and of the form $G / P$, where $G$ is a complex semisimple Lie group and $P$ is a parabolic subgroup.

I would like to thank Phillip Griffiths who suggested trying to find a short proof of Wang's theorem. I would also like to thank Professor Yozo Matsushima for his comments.

Proposition I. Let $X$ be a compact Kaehler manifold with $H^{1}(X, C)=0$, and let $S$ be a solvable connected complex Lie group acting holomorphically on $X$. Let $Y$ be a subvariety of $X$ invariant under $S$. Then $S$ has a fixed point on $Y$ and the fixed points form a subvariety.

Proof. First assume $Y$ is a manifold, $S$ is one dimensional and has no fixed points on $Y$. Associated to $S$ we have a holomorphic tangent field on $X$ and by invariance of $Y$ under $S$, also on $Y$; call it $A$. By assumption $A$ has no zeroes on $Y$.

We have short exact sequences where $\mathcal{O}_{Y}, \mathcal{O}_{X}$ are the holomorphic structure sheaves and $\Omega_{X}^{1}, \Omega_{Y}^{1}$ are the holomorphic one forms and $M$ is a subsheaf of $\mathcal{O}_{X} \cdot A$ and $\left.A\right|_{Y}$ as sections of the dual sheaves of $\Omega_{X}^{1}$ and

Received by the editors December 7, 1972.

AMS (MOS) subject classifications (1970). Primary 14C30, 14M15, 32J25, 32M05, $32 \mathrm{M} 10$.

Key words and phrases. Transcendental algebraic geometry and Hodge theory, homogeneous manifolds, automorphism groups of complex manifolds, Kaehler manifolds.

(c) American Mathematical Society 1973 
$\Omega_{Y}^{1}$ respectively give rise to natural maps denoted by the same letters. $r$ stands for the restriction map from $X$ to $Y$.

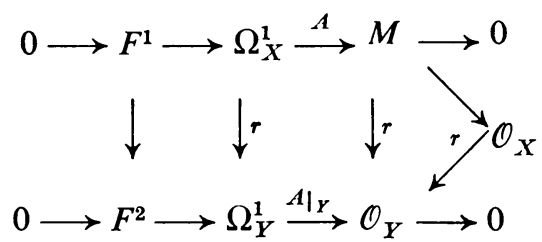

$A$ having no zeroes is equivalent to $\left.\Omega_{Y}^{1} \rightarrow{ }^{A}\right|_{Y} \mathcal{O}_{Y}$ being a surjection, and $F^{2}$ being locally free of rank $n-1$.

Passing to cohomology we have the commutative diagram:

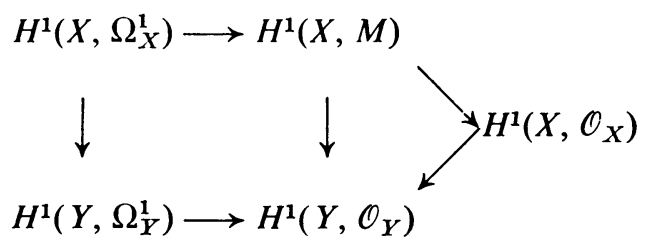

The Kaehler form $\omega$ on $X$ restricts to a Kaehler form $\left.\omega\right|_{Y}$ on $Y$. Since $H^{1}\left(X, \mathcal{O}_{X}\right)$ is a subgroup of $H^{1}(X, C)$ on a Kaehler manifold, it equals zero. Thus the image of $\left.\omega\right|_{Y}$ in $H^{1}\left(Y, \mathcal{O}_{Y}\right)$ is 0 and thus the Kaehler form of $Y$ is an element of $H^{1}\left(Y, F^{2}\right)$. By the Dolbeault isomorphism we can represent $\left.\omega\right|_{Y}$ by a $F^{2}$ valued 0,1 form. I observe that the $n$th exterior power of $\left.\omega\right|_{Y}$, where the dimension of $Y=n$, would be zero, since the fibre dimension of $F^{2}=n-1$. On the other hand the $n$th power of $\left.\omega\right|_{Y}$ is a nontrivial element of $H^{2 n}(Y, C)$, a volume form, which gives a contradiction.

We now remove the assumption that $Y$ is nonsingular. Simply note that $Y$ has a finite filtration by singular varieties

$$
Y=Y_{0} \supseteq Y_{1} \supseteq \cdots \supseteq Y_{m},
$$

where $Y_{s}$ is the singular set of $Y_{s-1}$. This filtration is respected by $S$ and the last element of the filtration is a manifold which we can take as $Y$.

The fixed point set is the zero set of $A$ and so is a subvariety.

Now let $S$ be of dimension $n$ and assume the proposition is true for $n-1$. Since $S$ is solvable it has a normal subgroup $S^{\prime}$ of dimension $n-1$. By assumption $S^{\prime}$ has a nontrivial fixed point variety $Y^{\prime}$ in $Y$. Note that $S$ leaves this new $Y^{\prime}$ invariant. To see this, take element $s$ of $S$, and an element $y$ of $Y^{\prime}$ and note that $S^{\prime}(s y)=s\left(S^{\prime} y\right)=s y$, so $s y$ is a fixed point of $S^{\prime}$ in $Y$, that is, an element of $Y^{\prime}$.

Pick an $A$ belonging to the complex Lie algebra of $S$ and not belonging 
to the Lie subalgebra of $S^{\prime}$. A gives rise to a one parameter subgroup of $T$ the universal cover of $S$, and hence to a tangent vector field on $Y^{\prime}$ and the fixed point set of $S$ in $Y$ is the fixed point set of $T$ in $Y^{\prime}$, and the first part of the proof applies. Q.E.D.

Proposition II. Let $X$ be a compact homogeneous Kaehler manifold with $H^{1}(X, C)=0$. Then $X$ is a projective manifold of the form $G / P$ where $G$, the connected component of the identity of $X$ 's complex Lie group of biholomorphic transformations is semisimple, and $P$ is parabolic, that is, contains a maximal solvable connected subgroup.

Proof. If $G$ were not semisimple it would have a solvable radical $N$. Let $x \in X$ be a fixed point of $N$ which exists by Proposition I. For all $g \in G$ we have $N(g x)=g(N x)=g x$. But $G$ is transitive and so $N$ leaves every point fixed and is thus the identity.

Let $B$ be a maximal solvable connected subgroup of $G$. $B$ has a fixed point $x$ and so $B$ belongs to the stabilizer of $x$, which we call $P$. Thus $x$ is of the form $G / P$ where $P$ is parabolic. Q.E.D.

REMARKS. It is easy to give simply connected complex manifolds where the Borel fixed point theorem is false, e.g. the Calabi-Eckmann manifolds.

The correct setting is probably on an appropriate generalization of Kaehler manifolds that one might call pseudo-Kaehler manifolds. These would be complex compact manifolds with a closed $C^{\infty} 1,1$ form which is positive definite on a Zariski open set. Hodge theory should go through for these manifolds, and then the above proof will be applicable.

The general structure of a compact Kaehlerian homogeneous space is given in [1].

The proof of the fixed point theorem above, looked at in the context of the Albanese map, actually proves a strong converse.

Proposition. Let $S$ be a complex connected solvable Lie group acting holomorphically on a compact Kaehler manifold X. S has a fixed point on any subvariety, including $X$, that $S$ leaves invariant if and only if the complex Lie algebra of holomorphic vector fields on $X$ associated to $S$ is annihilated by every holomorphic one form.

If $S$ has a fixed point, then the fixed point subvariety surjects onto the image of $X$ in its Albanese variety under the Albanese map.

In the case that there are no holomorphic one forms, that is $H^{1}(X, C)=0$, the above result reduces to the theorem in the paper.

A corollary of the above proposition is that a compact Kaehler manifold that possesses a nowhere vanishing holomorphic vector field $A$, also possesses a holomorphic one form $a$ such that $a(A) \neq 0$.

This proof and other related results will be presented in a future article. 


\section{BIBLIOGRAPHY}

1. A. Borel and R. Remmert, Über kompakte homogene Kählersche Männigfaltigkeiten, Math. Ann. 145 (1961/62), 429-439. MR 26 \#3088.

2. H. C. Wang, Closed manifolds with homogeneous complex structure, Amer. J. Math. 76 (1954), 1-32. MR 16, 518.

Department of Mathematics, Harvard University, Cambridge, Massachusetts 02138

Current address: Department of Mathematics, Yale University, New Haven, Connecticut 06520 